\title{
Sprachmaterial und Grammatik zum livländischen Livisch
}

<https://doi.org/10.33339/fuf.79544>

Salis-Livisch I. J. A. Sjögrens Manuskript. Ediert, glossiert und übersetzt von Eberhard Winkler und Karl Pajusalu. Veröffentlichungen der Societas Uralo-Altaica Band 88. Göttingen: Harrassowitz Verlag 2016. 392 S.

Salis-Livisch II. Grammatik und Wörterverzeichnis. Auf der Grundlage von J. A. Sjögren Sprachmaterialien verfasst von Eberhard Winkler und Karl Pajusalu. Veröffentlichungen der Societas Uralo-Altaica Band 89. Göttingen: Harrassowitz Verlag 2018. 403 S.

Das von J. A. Sjögren gesammelte und von F. J. Wiedemann herausgegebene Material der livischen Sprache (Wiedemann 1861a), das eine Grammatik und Sprachproben enthält, und das Wörterbuch des Livischen (Wiedemann 1861b), beide 1861 erschienen, sind eine einzigartige Quelle für die Erforschung des Livischen. Sie umfassen neben dem auch im 20. Jahrhundert in der Gemeinschaft gesprochenen Kurland-Livischen auch das im 19. Jahrhundert erloschene Salis-Livische. Die hier zu besprechenden Werke Salis-Livisch I und Salis-Livisch II im Folgenden der Einfachheit halber
SL I und SL II) sind ein wichtiger Beitrag zu diesem Komplex. SL I liefert eine Sprachprobe, die aus den Manuskripten Sjögrens besteht, aus denen Wiedemann (1861a) die gedruckte Sprachprobe zusammengestellt hat. SL II enthält eine Grammatik und ein Wörterverzeichnis, die auf der Grundlage dieser Texte erstellt wurde. Beide Verfasser haben sich eingehend mit der Erforschung des Livischen befasst. Eberhard Winkler hat alte livische Materialien in modernen Formen herausgegeben, darunter auch salis-livisches Material (Winkler 1994), und Karl Pajusalu hat die Phonologie und den Wortschatz des Livischen untersucht.

SL I umfasst 392 Seiten und enthält sechs Hauptkapitel. Kapitel 1 bietet einen Überblick über Sjögrens Manuskripte und deren Hintergrund. Es enthält drei eigene Hauptkapitel und bildet ein eigenständiges Ganzes. Das 1. Kapitel dieses Teils gibt Informationen über Sjögrens Livland-Reise (1.1), über die Informanten (1.2) und ihre Livischkenntnisse (1.3), über die Kommunikation zwischen Sjögren und den Informanten (1.4) und über Sjögrens Arbeitspensum (1.5). Kapitel 2 informiert über Aufbau und Inhalt der Manuskripte. In 
diesem Kapitel geht es einerseits um die äußere Gestalt der Manuskripte (2.1) und andererseits um inhaltliche Gesichtspunkte (2.2). Der letztere Teil ist weiter untergliedert in Wörterverzeichnis (2.2.1) und Sätze (2.2.2). Die Sätze werden in vier Unterkapiteln vorgestellt: Sätze ohne Vorlage (2.2.2.1) und solche mit Vorlage (2.2.2.2), Rätsel (2.2.2.3) und Übersetzungen von Bibelstellen (2.2.2.4). In Kapitel 3 wird dargestellt, was mit den Manuskripten geschah, als Wiedemann sie für den Druck redigierte. Zuerst werden die Teile von Wiedemann 1861a, die Salis-Livisches enthalten, in Form eines Inhaltsverzeichnisses vorgestellt; darauf folgt eine Tabelle, die anschaulich zeigt, welcher Teil des Manuskripts an welcher Stelle in Wiedemann 1861 vorkommt (3.1). Danach sind die Unterschiede in der Schreibung (3.2) und die Mängel der Wiedemannchen Edition (3.3) an der Reihe. Der letztere Teil gliedert sich in Fragen des Umfangs der Sprachprobe (3.3.1) und in inhaltliche Fragen (3.3.2).

Das Kapitel 1 enthält mit seinen den Text veranschaulichenden Abbildungen und Tabellen ein kompaktes Informationsmaterial über die von Sjögren gesammelten Materialien und ihre Hintergründe. Der Hintergrund der Manuskripte wurde gründlich untersucht. Auch diejenigen, die Wiedemann 1861a sorgfältig gelesen haben, sollten sich mit den gegebenen Informationen vertraut machen. Es handelt sich um Gesichtspunkte, die die Zuverlässigkeit des Materials betreffen. In ihrem Licht verdrängt SL I Wiedemann 1861a hinsichtlich der salis-livischen Sprachproben.

Kapitel 2 des Gesamtwerks präsentiert den Inhalt der Manuskripte insgesamt in heutiger Schrift. In Kapitel 3 werden die Texte des Kapitels 2 in Sätze, Satzgefüge und Phrasen geteilt und nummeriert sowie mit morphemweisen Übersetzungen, d.h. Glossen angeführt. Die deutschen Übersetzungen und Sjögrens kommentierende schwedische Übersetzungen stehen nebeneinander. Bei der Erstellung der Inhalte dieser Kapitel wurde gewaltige Arbeit geleistet. Schon die Elektronifizierung der Manuskripte in Kapitel 2 ist arbeitsaufwendig. Kapitel 3 enthält nach den Berechnungen des Rezensenten insgesamt 2156 Sätze, Satzgefüge und Phrasen, deren Glossierung gründliche Sachkenntnis erfordert. Zwar wurde der Grammatikteil SL II erst zwei Jahre später veröffentlicht, doch lässt bereits SL I die Materialkenntnis der Verfasser und ihre Sicherheit im Umgang mit grammatikalischen Fragen erkennen.

Die umfassende Analyse der grammatischen Einheiten enthält jedoch auch unklare Kommentare, 
die im später erschienenen SL II nicht erklärt werden. Auf diese komme ich im Zusammenhang mit SL II zurück.

Kapitel 4 enthält ein Verzeichnis neuer Wörter sowie derjenigen Wörter, die sich deutlich von dem Wörterbuch des Salis-Livischen unterscheiden, das die Verfasser früher herausgegeben haben (Winkler - Pajusalu 2009), Kapitel 5 verzeichnet die Abkürzungen und Kapitel 6 die Quellen.

Der Band SL II über Grammatik und Wortschatz umfasst 403 Seiten und besteht aus vier Hauptkapiteln: zur Orthografie (I), zur Phonologie (II), zur Morphologie und Morphosyntax (III), zu den syntaktischen Grundeigenschaften (IV), sowie zum Wortschatz in Sjögrens Manuskripten und dem Register. Diese Kapitel enthalten 4-5 eigene Unterkapitel, deren Nummerierung jeweils mit 1 beginnt. Der Band enthält ferner Korrekturen zu in SL I dargelegten Fragen, den Wortschatz in Sjögrens Manuskripten, ein deutschsprachiges Register des Wortschatzes, Anhang 1 Sprichwörter in salis-livischer Sprache, Anhang 2 ein kontrastives Wörterverzeichnis zu Sjögrens Manuskripten und älteren Quellen, Abkürzungen und Quellen. Für die Kapitel I und II sowie III 4.1 (die Unterkapitel 4.1.1-4.1.9 über die Verbstammtypen) zeichnet
Karl Pajusalu verantwortlich, für den Rest Eberhard Winkler (S. 14).

SL II on ein Musterbeispiel dafür, dass man in einer Grammatik nicht immer ein Sachregister benötigt. Die Inhalte der Kapitel I-IV sind mit ihren eigenen Hauptkapiteln und deren Unterkapiteln exakt nummeriert. Diese Kapitel enthalten insgesamt 19 Hauptkapitel, 68 Unterkapitel, 68 Unterkapitel der Unterkapitel und 56 Unterkapitel zu diesen. Durch diese Gliederung findet der Leser mühelos, was er sucht. Dass jedes Hauptkapitel wieder mit der Nummer 1 beginn, ist keine übliche Lösung, doch gerade sie schafft die Balance zwischen dem minutiös aufgegliederten Inhalt und einer vernünftigen Nummerierung. Auch im Hinblick auf den Seitenumfang der Grammatik - 174 Seiten - ist diese Lösung sinnvoll. Vor der Lektüre der Hauptkapitel kann man sich die Liste der Korrekturen zu SL I ansehen. So hat der Leser, der SL I durchgearbeitet hat, sofort Gelegenheit, Sachverhalte zu überprüfen.

Kapitel I bietet eine klare und kompakte Darstellung der Orthografie der Manuskripte Sjögrens und des vorliegenden Buches. In Kapitel II wird die Phonologie des Salis-Livischen unter intensiver Nutzung der in Sjögrens Manuskripten enthaltenen Wortfamilien dargestellt; dieses Kapitel erfüllt die informativen Anforderungen 
an eine Beschreibung der Phonologie in einer modernen Grammatik in jeder Hinsicht, von der Darstellungsweise bis zu den behandelten Themen. Viele Leser dürfte auch erfreuen, dass das Kapitel unter Berücksichtigung der gesamten livischen Sprache geschrieben ist, auch wenn die Darstellung als solche sich auf das Salis-Livische konzentriert.

Kapitel III über Morphologie und Morphosyntax beginnt mit den Nominalstammtypen. Die Darstellung schreitet vom sachlich und kompakt präsentierten Numerus zum Kasus fort. Hier beginnt eine aus der finnougristischen Grammatiktradition bekannte Lösung: Im Zusammenhang mit der Darstellung der morphologischen Elemente wird auch ihre Verwendung, d.h. die Morphosyntax dargestellt. Zum Anteil der Morphosyntax merkt der Verfasser warnend an, dass es sich um eine Aufzeichnung handelt und das Material für eine Darstellung der gesamten Grammatik nicht ausreicht (S. 80). In diesem Kapitel wird neben der Verwendung der morphologischen Elemente auch diejenige von Wörtern verschiedener Wortklassen dargestellt. Die Darstellung setzt sich bis ans Ende des Kapitels III so fort, dass sich die Morphologie jeder Wortklasse und ihre Morphosyntax abwechseln. Kapitel III enthält viele informative und nützliche
Tabellen und Verzeichnisse (S. 73, 77, 93, 98, 113-115, 131-132, 145-145, 150). Andererseits werden in eigenen Kapiteln Themen dargestellt, die keine Behandlung in separaten Kapiteln erfordert hätten und inhaltlich nicht mit dem Kapitel III harmonieren. Um zwei Beispiele anzuführen, seien Kapitel 1.4.3 über den Intensiv (S. 89) und Kapitel 2.6 über das Possessivpronomen (S. 101) genannt. Der erstere hat nichts mit der Morphologie zu tun, um die es in Kapitel III geht, und das letztere hätte im Zusammenhang mit den Personalpronomina behandelt werden können, wenn es nun einmal keine Possessivpronomina gibt.

In diesem Kapitel wird auch auf eine aus der Sicht der Grammatik recht bedeutungslose Frage eingegangen. Beispielsweise thematisieren die Verfasser auf Seite 85 die Verwendung des Terminus Dativ in der Dissertation des Unterzeichneten (Inaba 2015: 110-111); der Unterzeichnete hat auch für die Kasusform, die die Verfasser als Adessivallativ bezeichnen, den Terminus Dativ verwendet, mit der Begründung, dass in meinem salislivischen Material der größte Teil der Wörter im $l$-Kasus der Funktion nach habitiv sind, d.h. mit Belebtem verbunden. Es handelt sich um eine eher technische Entscheidung, die aufgrund der Frequenz von Wörtern im $l$-Kasus mit unterschiedlicher 
Funktion getroffen wurde und darauf zurückzuführen ist, dass der Unterzeichnete die $l$-Kasus des Salislivischen im Vergleich zum kurland-livischen Dativ untersucht hat. Auf eine solche Frage einzugehen, ist in einer grammatikalischen Darstellung völlig überflüssig, zumal die Verfasser selbst trotz ihres als umfangreich bezeichneten Beispielmaterials nicht wesentlich mehr Beispiele für die lokale Verwendung der $l$-Kasus vorlegen als der Unterzeichnete und einige dieser Beispiele nicht rein lokal sind (Beispiele (66) und (69)). Anstelle dieser terminologischen Problematik hätte man viele Punkte durch kurze Hinweise auf entsprechende Phänomene im Kurland-Livischen erhellen können. Einer dieser Punkte ist die dativische Verwendung des Genitivakkusativs der Personalpronomina (s. S. 82; 174-175). Ebenso hätten die Verfasser im Zusammenhang mit der dativischen Verwendung des Genitivakkusativs und den $l$-Kasus Angaben über die Häufigkeit der Belege machen können. So ist etwa das Beispiel (91) auf Seite 86 ood ome-lt sie pinn jära hüten.ImP2Sg Refl-ABL Dem.GA Hund.GA VPA 'Hüte dich vor dem Hunde' auch unter Einbeziehung der allgemein verfügbaren kurlandlivischen Korpora das einzige, in dem ein Belebtes bezeichnendes Wort in habitiver Funktion im Ablativ begegnet (s. Inaba 2015: 125-126).
Solche Informationen würden Licht auf die Repräsentativität der Beispiele werfen. Bei dem erwähnten Beispiel (S. 86) begnügen sich die Verfasser jedoch mit demselben verwunderten Kommentar wie bei dem betreffenden Satz auf Seite 216 von SL I. Kam ihnen nicht in den Sinn, dass es sich um semantische Äquivalenz handelt?

In Kapitel IV geht es um die syntaktischen Grundeigenschaften, womit Satztypen, Strukturen, grammatische Funktionen, Kongruenz und grammatische Funktionen des Satzes gemeint sind. Gleich zu Beginn wird vor drei Faktoren gewarnt, die in diesem Kapitel eine Rolle spielen: 1) Sjögren sprach weder lettisch noch livisch, so dass er die Texte mithilfe eines Dolmetschers erhielt, der des Lettischen mächtig war, 2) die Texte sind mehr oder minder Übersetzungen, 3) die sprachliche Kompetenz zweier Informanten war offenbar geschwächt, denn sie berichteten von Gesprächen mit anderen Sprechern des Livischen.

In diesem Kapitel ist die Anordnung der behandelten Themen recht verworren. Beispielsweise werden in Kapitel 2 die zu den sog. Basissatztypen gehörenden Themen, wie Prädikativsatz, Possessivsatz und Existentialsatz, auf der gleichen Ebene behandelt wie die anhand der Nominalformen des Verbs gebildeten Konstruktionen. Bei der Lektüre des 
Kapitels mag beim Leser die Frage aufkommen, aus welchem Grund die einzelnen Themen zur Behandlung ausgewählt wurden und an wen sich die Darstellung richtet. Dies liegt teilweise daran, dass in der Grammatik einige Termini benutzt werden, deren Verwendung als Kapitelüberschrift zumindest im finnougristischen Kontext ungewöhnlich ist. Hierzu gehören das direkte und indirekte Objekt (S. $171 \$ 4.3 .1$ Direktes Objekt und S. $174 \$ 4.3 .2$ Indirektes Objekt; s. auch S. 80 und S. 85). Eine solche terminologische Lösung kennt man aus Grammatiken derjenigen germanischen Sprachen, deren Kasussystem sich vereinfacht hat, so dass ein und dieselbe Form je nach der Position sowohl das Akkusativ- als auch das Dativobjekt vertritt. Im SalisLivischen betrifft eine vergleichbare Situation nur die Genitiv-AkkusativFormen der Personalpronomina (im Singular), während Substantive in dativischer Funktion mit dem Allativadessiv markiert werden, der nicht zur Markierung des (Akkusativ)objekts verwendet wird. Daher bleibt der Grund für die Entscheidung in SL II unklar und man kann den Eindruck gewinnen, dass die salis-livischen Entsprechungen anhand der Grammatik einer der erwähnten germanischen Sprachen dargestellt wurden. Unklar bleibt auch, aus welchem Grund bei den anderen Komplementen nur die elativischen, illativischen und translativischen Satzglieder einbezogen wurden (4.3.3, S. 175-177). Andererseits enthält das Kapitel 2.2 (S. 137) über das Prädikativum nur Sätze mit dem Verb old. Ein Teil der zu dieser Kategorie gehörenden Sätze (s. Viitso 2008: 342; Inaba 2015: 153-167) wird in Kapitel 2.6 über das Futur behandelt (S. 160). Hinsichtlich der in der Grammatik zu behandelnden Themen und der zu verwendenden Termini hätte man sich an den Grammatiken der mit dem Livischen nahe verwandten Sprachen Estnisch und Finnisch oder direkt an den Beschreibungen des Kurland-Livischen (z.B. Viitso 2008: 315-345) orientieren können. In diesem Kapitel, wie auch in Kapitel III, hätte man die früheren Untersuchungen zur livischen Sprache durch Verweise stärker nutzen können. Die Verweise sind jedoch minimal.

Die erwähnten Mängel beeinträchtigen das Verständnis nicht. Im Hinblick auf die Kommentare zu den Sätzen in SL I hätte man jedoch gerade von der Grammatik in SL II Präzisierungen erwartet. Um ein Beispiel zu erwähnen: In SL I steht auf Seite 320 der Satz tä- $d-l$ vajag puude-l oll ihr-PL-ADAL nötig rein-AdAL sein.INF 'Ihr müsst reinlich sein'. Im Kommentar zu diesem Satz heißt es, die (Kasus)kongruenz des Infinitiv-Subjekts tädl und des Infinitiv-Prädikativs puudel entspreche wahrscheinlich dem Muster 
des Lettischen. Ein ähnlicher Kommentar wird auf S. 326 zu dem wort rikkel in dem Satz mis sie ääbt-is rikke-l oll? IPR DEM helfen-PRÄT reichAdAl sein.InF 'Was half es, reich zu sein?' gegeben und betont, dass es sich nicht um echtes Livisch handelt. Gerade zu solchen Kommentaren hätte man in der Grammatik in SL II Präzisierungen erwartet, die jedoch nicht gegeben werden. Das Thema wird weder in dem Kapitel über die Verwendung des Adessivallativs noch im Kapitel über das Prädikativum erwähnt (s. S. 84-86; 157 \$2.2; vgl. Inaba 2015: 153-167).

Die Kapitel III und IV enthalten einige Fehler hinsichtlich der Beispiele, die im Widerspruch zu in denselben Kapiteln dargestellten Sachverhalten stehen. Um zwei zu erwähnen: Bei dem Beispiel (29) auf Seite 82 ist bei dem genetivakkusativischen Satzglied nicht von Inkongruenz, sondern von Halbkongruenz zu sprechen (s. S. 182). Die Worte sinn und tänn in den Beispielen (713), (715) und (716) auf Seite 175 (s. auch S. 176 Beispiel (722)) sind nicht illativisch, sondern lativische Formen von Adverbien mit unvollständigem Paradigma, die nur von ihrer Funktion her den illativischen Formen der Substantive entsprechen (s. 149).

Der Wortschatz der Manuskripte Sjögrens ist klar dargestellt und angenehm gesetzt. In vielen
Wortartikeln werden die Varianten der Belege übersichtlich nach morphologischen Kategorien gegliedert. Man kann das Wörterverzeichnis also nutzen, um die konkreten Belege der Wörter zu klären, ohne die Sätze in SL I zu lesen. Auch das Register zum Wortschatz (S. 333-355) gibt Aufschluss darüber, welche Wörter in den Manuskripten begegnen.

Der erste der beiden Anhänge enthält 36 Sprichwörter in salis-livischer Sprache, die dem von Mälk et al. herausgegebenen Werk entnommen wurden. Das kontrastive Wörterverzeichnis der Manuskripte Sjögrens und anderer alter Quellen in Anhang 2 informiert übersichtlich darüber, welche Wörter der Manuskripte in welcher Quelle und in welcher Form begegnen.

SL I und II bilden eine hervorragend verwirklichte Gesamtheit, die von der Sprachprobe bis zur Grammatik die lückenhaften und verfälschten Informationen über das Salis-Livische ergänzt und korrigiert, die u.a. in Wiedemann 1861a gegeben werden. Die Erstellung der Bände hat selbst bei Verwendung elektronischer Texte einen enormen Arbeitsaufwand erfordert. Die Bedeutung des Resultats dieser Arbeit für alle, die sich mit der Erforschung des Livischen beschäftigen, ist unbestreitbar, und SL I und II werden zweifellos in ihrem Kreis 
breite Verwendung finden. Andererseits weckt u.a. SL II trotz der gelungenen Gesamtheit eine ironische Frage: Wäre eine allein auf das Salisch-Livische konzentrierte Grammatik nötig gewesen? Wer braucht so etwas? Aufgrund des geringen Umfangs des erhaltenen Materials liegt die größte Bedeutung des Salisch-Livischen im Vergleich mit dem Kurland-Livischen als Teil der livischen Sprache und in der Gesamtheit der ostseefinnischen Sprachen, d.h. im diachronen Kontext des Ostseefinnischen. Insofern hätte der grammatische Teil von SL II mit einem etwas einheitlicheren Ansatz geplant und verwirklicht werden können. Wie bereits erwähnt, berücksichtigt das Kapitel über die Phonologie das Kurland-Livische und unterscheidet sich damit von den späteren Kapiteln der Grammatik. Auch in den späteren Kapiteln ist die Rede vom Estnischen, Finnischen, Kurland-Livischen und Lettischen, aber die Erwähnungen sind $\mathrm{zu}$ einem gewissen Grad isoliert, was vermutlich darauf zurückzuführen ist, dass die Darstellungsweise von der finnougristischen und aus den Grammatiken der ostseefinnischen Sprachen bekannten Tradition abweicht. Insofern entsteht kein Bild von der livischen Sprache als Ganzem. Vieles hätte durch kurze Erwähnungen entsprechender Sachverhalte im Kurland-Livischen und Verweise auf Untersuchungen und Darstellungen des Kurland-Livischen erhellt werden können. Für eine solche diachrone Herangehensweise hätte Wiedemann 1861a als Muster dienen können.

Nobufumi Inaba

\section{Quellen}

INABA, Nobufumi 2015: Suomen datiivigenetiivin juuret vertailevan menetelmän valossa. Suomalais-Ugrilaisen Seuran Toimituksia 272. Helsinki: Suomalais-Ugrilainen Seura.

MäLK, VAINA (ed.) 1981: Liivi vanasõnad eesti, vadja ja läti vastetega. I-II. Koostanud Vaina Mälk, Pētõr Dambergi, Elza Kokare, Arvo Krikmanni, Lembit Vaba, Tiit-Rein Viitso, Eduard Vääri osavõtul. Tallinn.

Virtso, Tirt-Rein 2008: Liivi keel ja läänemeresoome keelemaastikud. Eesti Keele Sihtasutus, Tartu - Tallinn.

Wiedemann, Ferdinand Johann 1861a: Joh. Andreas Sjögren's Gesammelte Schriften. Band II. Theil I. Livische Grammatik nebst Sprachproben. St. Petersburg: Eggers et Comp.

Wiedemann, Ferdinand Johann 1861b: Joh. Andreas Sjögren's Gesammelte Schriften. Band II. Theil II. Livischdeutsches und deutsch-livisches Wörterbuch. St. Petersburg: Eggers et Comp.

Winkler, Eberhard \& Karl Pajusalu (eds) 2009: Salis-Livisches Wörterbuch. Teaduse Akadeemia Kirjastus, Tallin.

Winkler, Eberhard 1994: Salis-Livische Sprachmaterialien. Veröffentlichungen des Finnisch-Ugrischen Seminars an der Universität München. Serie C: Band 21. München. 